\title{
Gels for constant and smart delivery of insulin
}

\author{
M JOAN TAYLOR, KRISHAN P CHAUHAN, TARSEM S SAHOTA
}

\begin{abstract}
The focus of this review is the role of gelatinous materials for oral, transdermal and peritoneal insulin platforms as alternatives to the ubiquitous subcutaneous depot approach. Hydrogels that form hydrated, cohesive materials and the topologically complex micellar types can add ligand interaction, bond vulnerability and rheological characteristics to develop reliable programmed release, including closed loop (automated basal and bolus) activity in non-oral routes. In addition, the potential protection of the protein and likely increased paracellular uptake mean that orally active insulin is feasible. While unlikely to be suitable for closed loop delivery, the driver for gut absorption is not only to increase the convenience and decrease dosage trauma, but to target the mesentery-portal vasculature rather than peripheral tissue, thus improving hepatic glycogen equilibrium and reducing the obesogenic effect and hypoglycaemic episodes. Br J Diabetes 2020;20:41-51
\end{abstract}

Key words: insulin, drug delivery, polymer, gel, hydrogel, micelle, critical solution temperature, rheology, visco-elastic, closed loop, kinetic, enzyme protection, ligand, interactive, oral, subcutaneous, peritoneal

\section{Introduction}

The discovery of insulin in 1921 and its rapid introduction as mainstream treatment for type 1 diabetes (T1D) was truly lifesaving. Until then, anyone developing T1D faced practically certain death. ${ }^{1}$ However, it is well recognised - almost 100 years later - that, despite many improvements in the tools for assessing blood glucose $(B G)$ and administering insulin, those who need it for either T1D or type 2 diabetes (T2D) still run the risk of developing the complications of diabetes. Landmark randomised trials have underlined the need for a much more tailored approach to insulin therapy for both conditions, ${ }^{2,3}$ with more frequent insulin doses correlated where possible to the ambient BG, the carbohydrate content of an impending intended meal and the likely forthcoming energy expenditure.

School of Pharmacy, De Montfort University, Leicester, UK

Address for correspondence: Professor M Joan Taylor School of Pharmacy, De Montfort University, Leicester LE1 9BH, UK E-mail:mjt@dmu.ac.uk

https://doi.org/10.15277/bjd.2020.248
Intensive insulin therapy is demanding but has become the standard for T1D, albeit with many daily finger-sticks and the everpresent hazard of hypoglycaemia. The debate beyond T1D, in extending tight control to T2D insulin users, is interesting with many care providers advocating the upwards titration of insulin to better control $\mathrm{BG}$, but a problem being that this is potentially obesogenic $c^{4,5}$ in addition to the hypoglycaemic risk.

Parenteral insulin is not an easy regimen to manage, ${ }^{6}$ whether by pen injection, patch pump or conventional pump. New insulin types, sensor technology and phone apps have improved matters, but patient acceptability, compliance and kinetic considerations are important reasons for developing the other paradigms. These are discussed here in the context of gel formulations that could be used as such by various routes or inside a device. Gels offer compatibility with insulin and tissue, enhanced uptake by tissue and protection from enzymic destruction. There is also the possibility of kinetic control to produce not only continuous dosing at a constant background rate but also combination with routes that could provide hepatocentric strategies. The latter mimic the physiological norm and would minimise the obesogenic effects of insulin. In addition, automated glucose-responsive bolus doses could be delivered by designer gels, especially where using fast administration routes would allow real-time transmission of the altered insulin dose rate, thus producing triggered and closed loop systems that could obviate hypoglycaemia. The structure and properties of gels potentially able to deliver on these ideas are discussed below.

\section{Suitability and biocompatibility of gel carriers}

Soluble insulins, whether native or biosynthetic monomeric types, are charged polar molecules. ${ }^{7}$ Consequently, they are water soluble although this is $\mathrm{pH}$ - and additive-dependent. Suitable candidate carriers for insulin delivery via the routes mentioned are therefore also often highly hydrophilic, arising from natural and synthetic sources. Water-soluble, dispersible or swellable gels feature strongly in the literature with impressive inventions for solving the problems of diabetes treatment, including by closed loop. Hydrophobic and dried (xero) gels exist, but this review section will focus on gelling systems with which insulin is likely to interact, using various types of carrier linking and mechanisms built in to achieve the goals of dependable but flexible dosing. Hydrophilic gel types are additionally appropriate because of biocompatibility, especially if of natural origin such as chitosan, heparin, casein, pectin, alginate, tragacanth, dextran and gelatin-based materials. ${ }^{8}$ Ultimately, tissue response 
Figure 1. Simple gels and the incorporation of second components that bond around the original structure to form interpenetrating gels; both types are used to develop materials for drug delivery

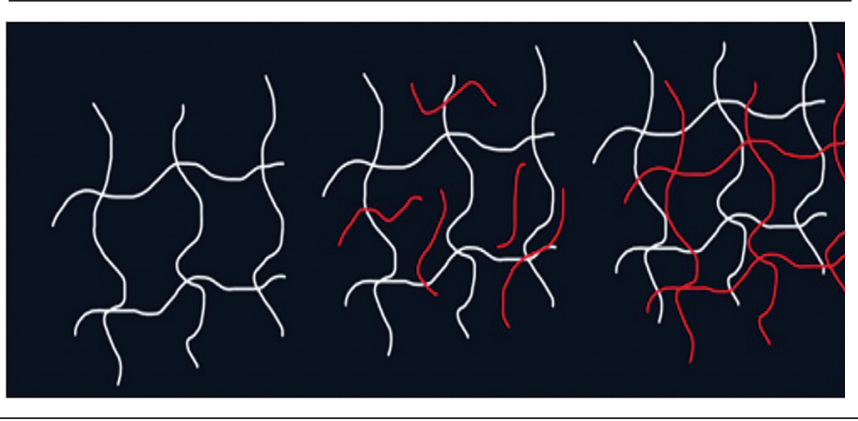

depends, however, on many demanding factors and some synthetics provide properties that are useful (eg, cyclodextrin, acrylics, etc). Some gels are bio- and/or muco-adhesive, a property that can overlap with viscosity but differs from it and can be used to extend residence time in, for example, the gut. ${ }^{8}$ Intense research has led to materials that are capable of forming safe oral, parenteral, implantable or mucous membrane delivery devices for insulin basal and bolus dosing, ${ }^{9}$ although none has yet reached the clinic.

\section{Gel structure}

A gel is typically described as a semi-solid, often with the continuous permeation of at least one three-dimensional structure (the gelator system) with a second (normally a liquid 'solvent'). Where more than one gelator is present, there may be a synthesised interlacing of solvated polymer frameworks that precludes separation unless bonds are broken; this is known as an interpenetrating network (IPN), discussed under hydrogels (Figure 1). Gels may be simple or smart with ligand interaction for differential delivery.

\section{Gels designed to deliver co-formulated peptides}

The entrapping of co-formulated peptides such as insulin in the network depends on specific polymer characteristics and the gelling mechanism. The gelator may produce simple entanglements, especially if branched. Alternatively, there may be physical interlocking because of attractive forces acting between specialised regions (see Release mechanism: 2 - Bolus below) or there may be covalent bonding (see Hydrogels below).

Some gels are not homogeneous but are formed by the combination of particulates including a range of micro- and nanostructures such as minigels, micelles and liposomes (see below). Each hydrated gel type produces internal solvent-filled spaces potentially on the scale of the peptide inclusions that are present either as molecules dissolved in the solvent phase or as much larger undissolved drug particles where the displaced polymer network forms around them.

The polymeric gelators are usually in lower concentration than the solvent and can be similar to materials routinely used
Figure 2. (a) Parallel plates used in oscillatory mode, (b) the imposed strain and response signals, and (c) the captured parameter data translated into moduli and tan delta plots

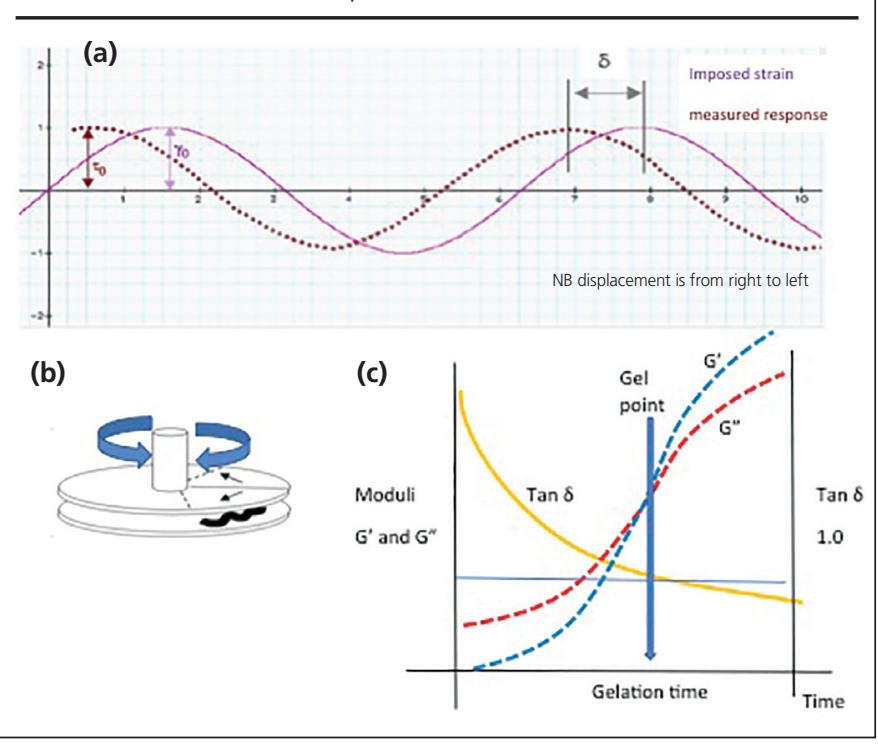

to increase the consistency of liquid pharmaceuticals and foods - for example, as suspending and emulsifying agents like polyacrylic acid. The technical specification that separates mere thickening in consistency from true gelling is revealed in viscoelasticity measurements (Figure 2), so that a gel has a value of less than unity for tan delta (or damping factor) which is the ratio of the loss modulus, G", to the elastic modulus, $G^{\prime} .{ }^{10}$ In descriptive terms, this means that the loss modulus becomes less prominent than the elastic counterpart as a system gels. The perfectly elastic solid would return the imposed shear signal synchronously and thus any shift (the phase angle delta, for which the tangent is normally stated) represents the $G^{\prime \prime}$ energy loss both as heat and viscous flow from the theoretical perfect elastic response (the elastic modulus).

Relevant properties are typically measured by oscillatory rheometry using a stress amplitude that is within the viscoelastic limit for the material concerned, so that with the almost imperceptible oscillation, the structure remains undamaged throughout the stress testing. Typical oscillation tests involve changes with time, shear rate frequency or temperature sweeps and can reveal critical tan delta values occurring as the physical chemistry changes to allow the elastic modulus to dominate or fade. Rheometry is a powerful tool to hone the gel behaviour for relevance to insulin drug delivery because the viscous component relates to the likelihood of drug diffusion, assuming the pore size is appropriate.

\section{Hysteresis}

In some gels, such as agarose and gelatin, the physical stabilisation arises on cooling from interlocking helical segments in otherwise linear polymeric chains (Figure 3a). Repeated cycles for these often give characteristic hysteresis loops if the complex 
Figure 3. (a) The structures formed as gelatin cools. (b) Plotted modulus graphs showing hysteresis

(a)

$$
\begin{aligned}
& \text { single helix sections } \\
& \text { superimposing to } \\
& \text { form triple helical } \\
& \text { crystalline regions }
\end{aligned}
$$

random coil sections (amorphous) (b)

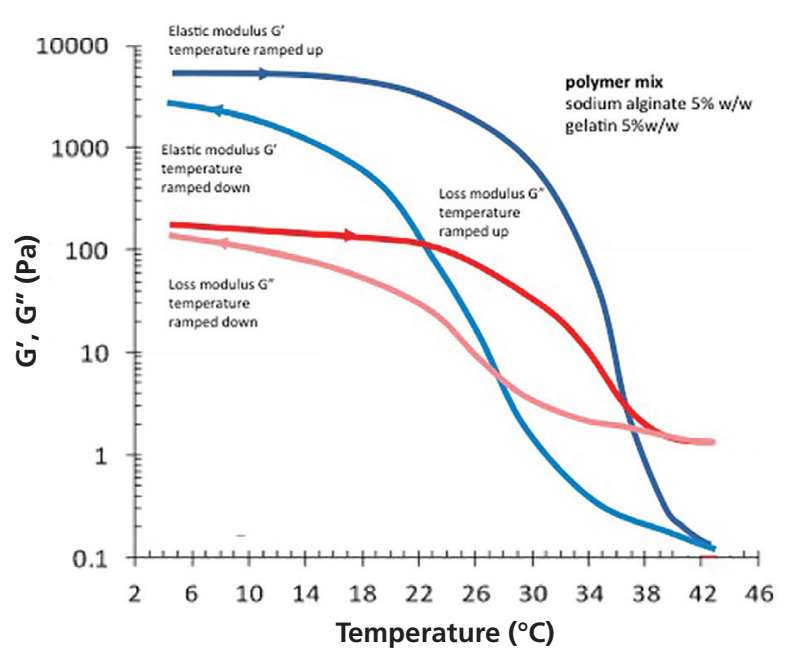

structures reform more slowly than the test cycle time (Figure 3b). Hysteresis creates uncertainty in the gel performance for drug delivery but, in any case, gel formulations that lose consistency at body heat are obviously of limited use for insulin delivery, since they are neither injectable (unless hot) nor are they gelled at body temperature. Even so, these agents are biocompatible and have often featured in more complex formulations.

\section{Innovative design using gelling polymers}

Synthetic co-polymers comprise combinations of monomers, synthetic or otherwise, that can be incorporated in linear or branched, regular or random patterns, including grafted and block types (Figure 4). These have been exploited for materials useful for convenience of administration, controlled release, specific responses and target recognition, all of which have a place in the delivery of insulin, other peptides or drug molecules in general. ${ }^{11,12}$

Figure 4. Various polymeric types that form gels with properties determined by monomeric inclusion and morphology

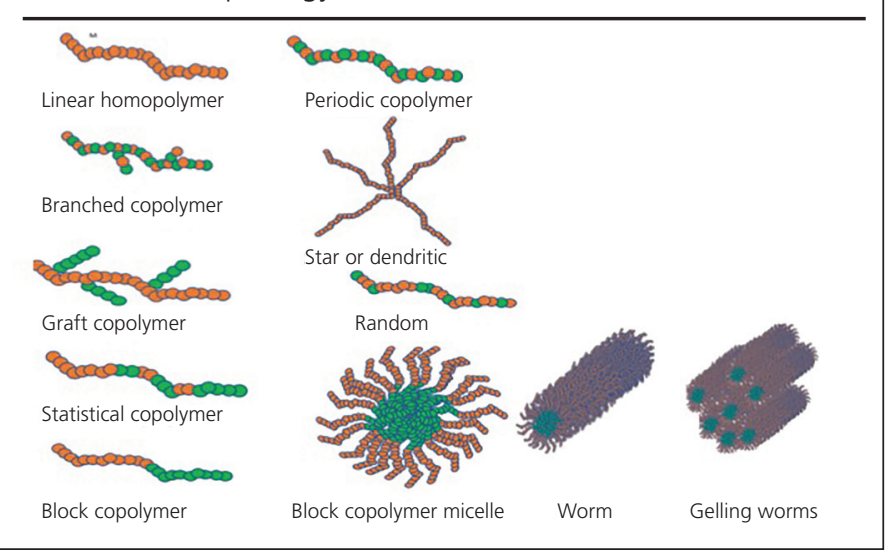

Block polymers may micellise and group to create gelling phenomena that may change with ambient conditions (Figure 4). Insulin can be carried within or between the structures. One special effect is reverse thermogelling, as with the well-known poloxamer family (eg Pluronic ${ }^{\odot}$ ). Poloxamers have polyoxyethylene (POE) and polyoxypropylene (PPO) sections as a POE-PPOPOE sandwich that, in appropriate concentration, cause gelling as the single molecules micellise in an entropically-driven selfassociation of hydrophobic moieties as temperature increases above a critical level. ${ }^{13}$

Figure 5. Upper and lower critical solution temperature plots (Taylor, 2017)

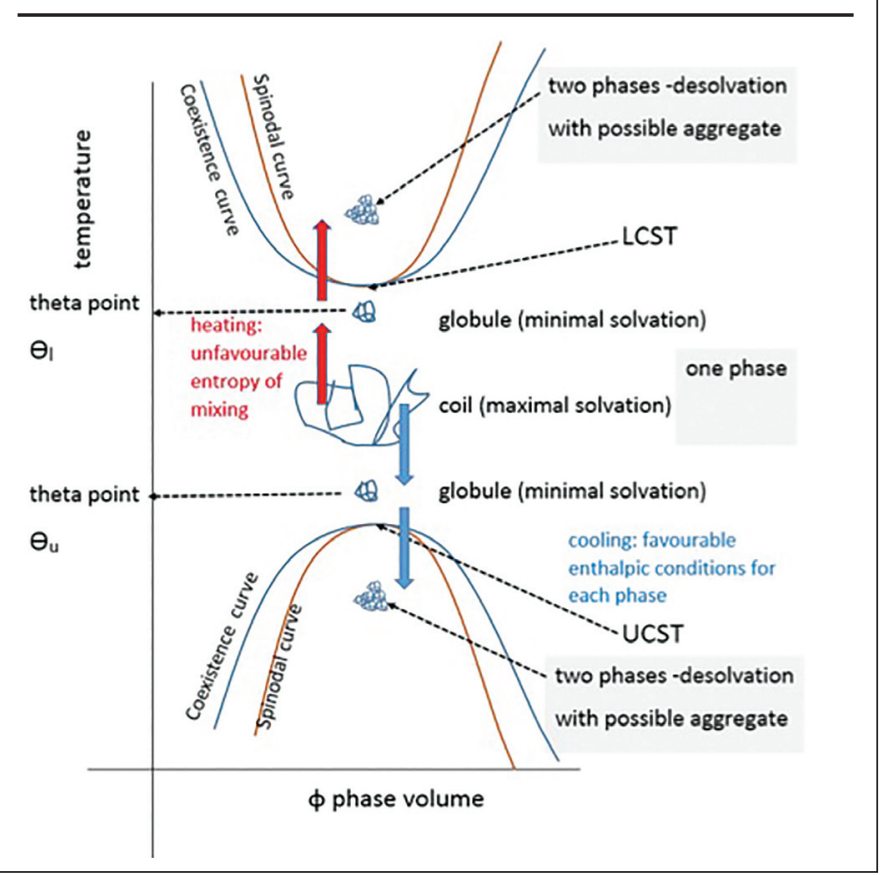


This broadly fits with the coil to globule shrinkage as the structure approaches critical solution temperatures. The lower critical solution temperature (LCST) characterises the behaviours of reverse gelling materials (ie, those that gel on heating rather than cooling) (Figure 5)..$^{14}$

Pluronic $\mathrm{F}-127^{\circ}$ is liquid when refrigerated but, at suitable concentration, gels above about $20^{\circ} \mathrm{C}$ (depending on purity). It is therefore solidified at body temperature, making it relevant for in situ gel formation deliverable as a cold injection. Choi and Kim used a more persistent gelling polyester-POE-polyester ( $\gamma$ PGLA-POE- $\gamma$ PGLA) synthetic to deliver insulin in rats, ${ }^{15}$ but Chen et al described a similar system that may have relevance for detemir. They examined both the physicochemical characteristics and the release in mice ${ }^{16}$ and confirmed the formation of core-corona micelles, but which in this case incorporated liraglutide into this structure because of its own amphiphilicity rather than randomly as a passive passenger where it might otherwise be held within or between micelles in the analogous poloxamer. This allowed lengthy sustained release and also degradation to non-toxic products.

Micelles interact with tissue features such as tight junctions in a manner crucially different from that of larger particles, but they also can transport insulin across membranes. The incorporation of recognition ligands such as transferrin, niacin, lectins and antibodies or cell-penetrating peptides such as penetratin make a variety of effects possible for the specialised delivery of insulin ${ }^{17}$ (see also Figure 8).

\section{Hydrogels}

A different type of gel system occurs where stabilisation occurs between gelator linear polymers. This is mediated mainly by covalent links, but gelatin is a physically stabilised hydrogel, as discussed above. The cross linking agents can be double or multivalent in terms of the connections they make and can therefore also be defined as gelators, imposing three-dimensional structure on linear systems that might be viscous but would not otherwise technically gel. These can also be interpenetrating gelators, as discussed earlier (Figure 1). The resulting materials are hydrogels with the cross link density influencing the rigidity and the pore size either in bulk or in gelatinous particulates (see Minigels below). ${ }^{18,19}$

Hydrogels have been formed from a variety of natural and synthetic materials including the innovative star-PEG gelated heparin, as shown in Figure 6. As with micellar gels, smart ligands can be designed to create responsive materials.

Hydrogels are insoluble because of their very high molecular weight, although often soft and pliable when hydrated. In that state they have interesting condition-dependent shrink-swell and phase change characteristics where the analogous non-cross linked materials have differential solubility instead. ${ }^{20}$ Some materials can be injected to form covalently stabilised hydrogels in situ by, for example, enzymatic coupling. ${ }^{21}$ Nguyen et al have recently described the in situ hydrogel formation of novel pentablock copolymer sols, where insulin was released from coformulated chitosan inclusions. ${ }^{22}$ Photo-cross linked types have
Figure 6. Heparin, a polymerised aminosaccharide converted to three-dimensional gel, using star-PEG

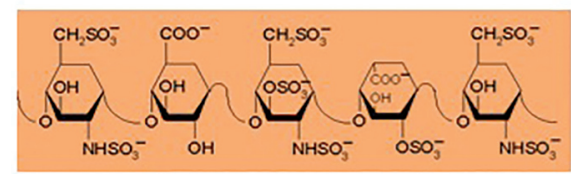

Heparin

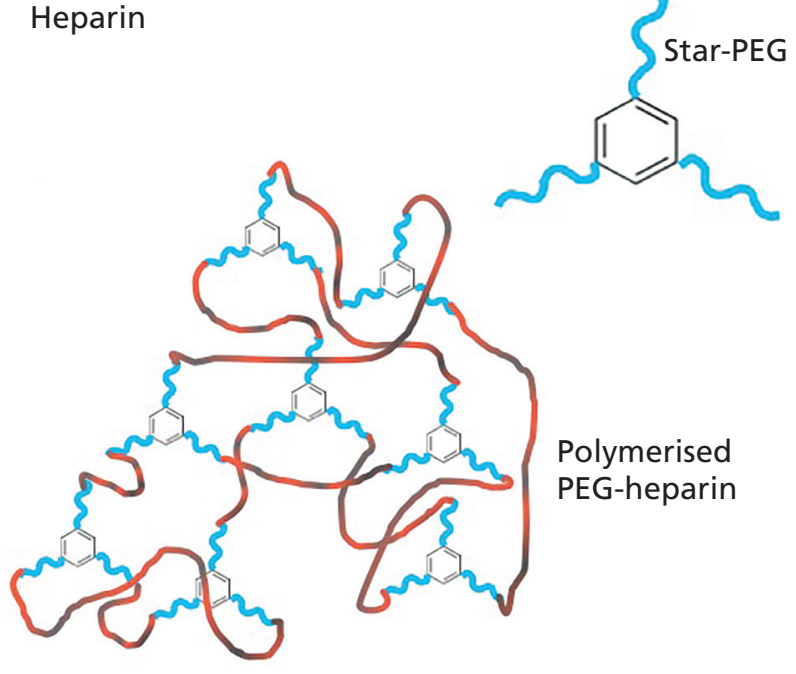

also been suggested, such as that described by Custodio et al for hydrogels formed from laminarin, a low molecular weight glucan..$^{23}$ Some can be placed subcutaneously as dried pellets to hydrate in situ and deliver peptides such as insulin long term. Disadvantages are that any implant into tissue could be immunogenic and attract fibrous growth, can contain toxic monomers, may harbour infection or invite opportunist blood-borne infection. ${ }^{24-26}$ If not safely metabolised, they may also need surgical removal and thus be unsuitable for implanted insulin delivery.

However, the same types of materials can be formulated for other routes of administration such as oral, other mucous membrane or transdermal. Minigels (particulate hydrogels) present a useful vehicle in this regard. They are closely packed, globular hydrates that give the tactile and visual impression of a confluent gel. An example is polycarboxylic acid polymers of various types (carbomers, eg $\left(\right.$ arbopols $\left.^{\odot}\right)$. Some versions of these are cross linked with allyl sucrose, comprising particles of about $0.2 \mu \mathrm{m}$ diameter and a molecular weight of several billion. They flocculate to a diameter of up to $7 \mu \mathrm{m}$, but when hydrated may approach $70 \mu \mathrm{m}$.

These particles can carry molecular cargo within the aqueous pores produced by the cross links ${ }^{27}$ if the drug molecule is small enough. Larger molecular weight agents can certainly be carried between the hydrated particles (minigels) and this space can also accommodate undissolved finely divided solid. For insulin, the latter could be crystalline or amorphous precipitate, provoked by high concentration or $\mathrm{pH}$. The addition of protamine or excess zinc also causes loss of insulin solubility and could be accomplished in these gels. The viscosity of such a gel is compli- 


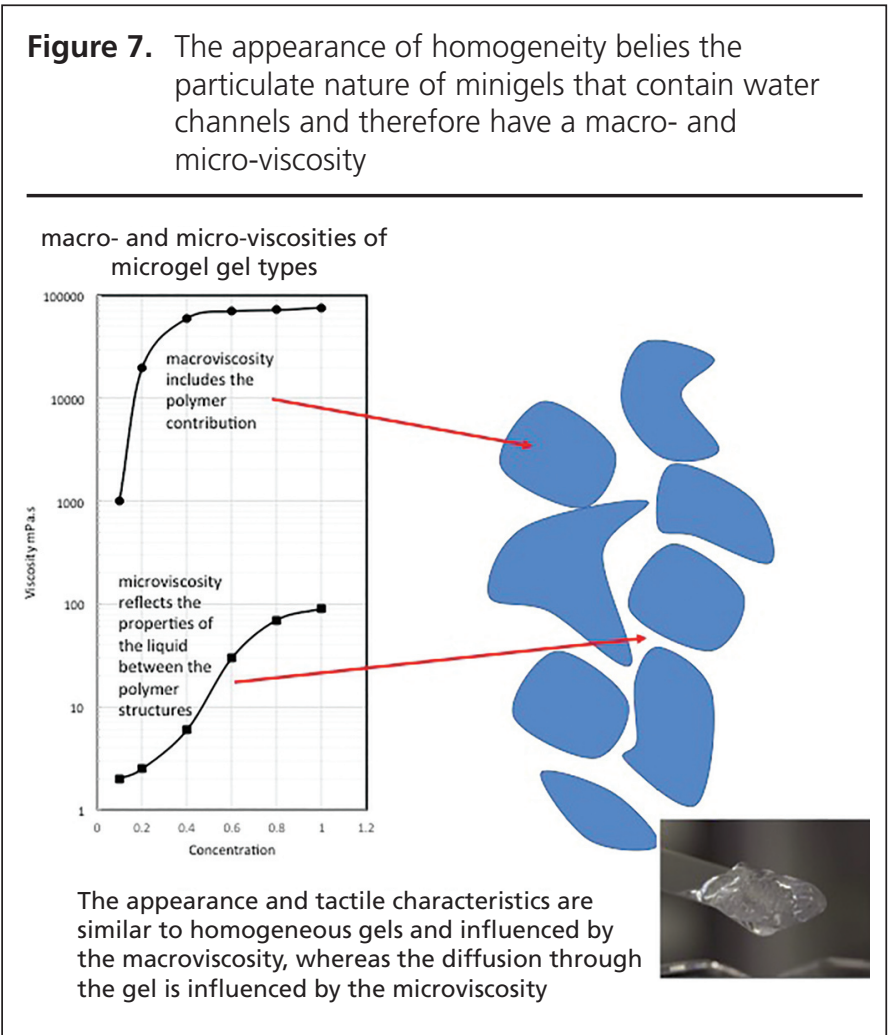

cated by the dual nature of the material having an overall consistency as measured by rheometry but a microviscosity of the channels between minigels, in which the drug diffusion is more likely (Figure 7). Reverse micelle imprinting can selectively trap structures such as insulin, ${ }^{28}$ and interactive ligands ${ }^{29}$ that respond to glucose by a rheological change are also possible.

Smaller entities such as micro- and nano-particles (1-100 $\mathrm{nm})$, including nano-sized micelles with their ability to access tight junctions, have been fabricated to carry insulin (Figure 8). Nanoparticles can be gelatinous because of their polymeric form but, in any case, their surface energy density differs from that of larger entities ${ }^{30}$ and they potentially overcome barriers such as hostile gut environments and apical impermeability. ${ }^{17,31}$ This

Figure 8. Transport across membranes of simple and ligand-bearing micelles released from micelle gels

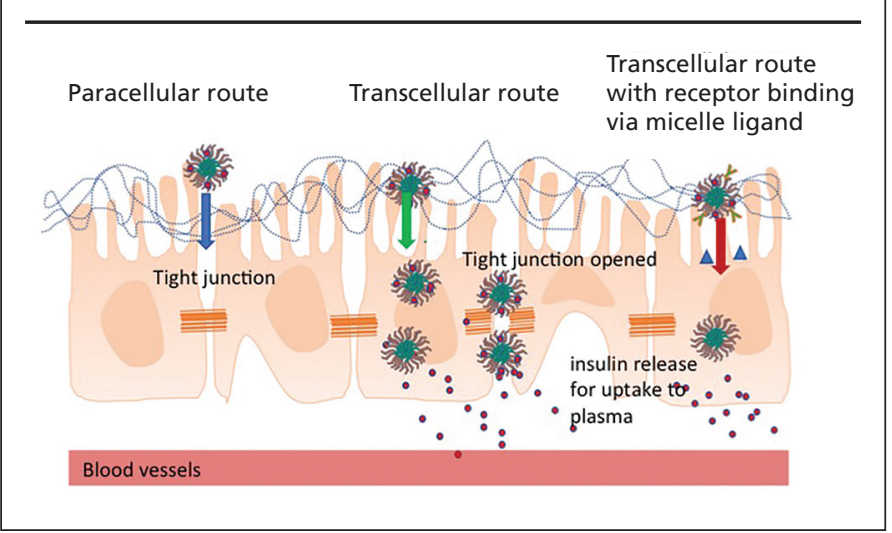

latter advantage is important because of the mesenteric drainage to the portal system. The first pass effect associated with oral delivery is doubly useful because the dose is short lived, which may mitigate hypoglycaemic threats, but also a major antihyperglycaemic effect is the polymerisation of glucose to glycogen in the liver. Subcutaneous insulin produces a nonphysiological ratio of peripheral to hepatic effects by comparison that can promote the unwanted accumulation of fat. ${ }^{32}$

Nano-structures vary considerably. Unlike micelles, many are particles that are not self-assembling and, apart from size, have more in common with both minigels and microparticles. The composition varies from natural polymers such as chitosan, alginate, dextran, hyaluronic acid and other polysaccharides, ${ }^{33-36}$ but also includes synthetically altered chitosan such as carboxylated, trimethylated (TMC), ethyl (DMEC), mucin-complexed, ${ }^{37}$ carboxymethyl (CMCS), oleic acid-zinc insulin complexed, ${ }^{38}$ thiolated chitosan ${ }^{39}$ and vitamin B12-conjugated chitosan (VitB12Chi) among many others. Chaturvedi et al included a complex multicomponent particle designed to optimise gut absorption. ${ }^{9}$ Synthetics such as poly( $\gamma$-glutamic acid) $(\gamma-P G A)$, phospholipid $\gamma$-PGA conjugates and polyacrylics such as the various Eudragit ${ }^{\odot}$ materials have also been used. Some of these are built layer by layer to achieve particular goals (as discussed below in the context of glucose sensitivity) ${ }^{40}$ as are further micellar examples and the related liposomal structures.

Superficially similar to micelles are liposomes (Figure 9), which are uni- or multi-lamellar vesicles that self-assemble when phospholipids of various types, sometimes co-formulated with

Figure 9. Liposomes in context with micelles. The micelle is much smaller and formed from block polymers in this case (differing from surfactant micelles) while the liposome is formed from lipid bilayers with polar head groups (orange) on the inner and outer aspects, the lipid chains in the interior of the double layer. Liposomes can comprise one or many concentric bilayers. Both micelles and liposomes can form tubular gelling structures
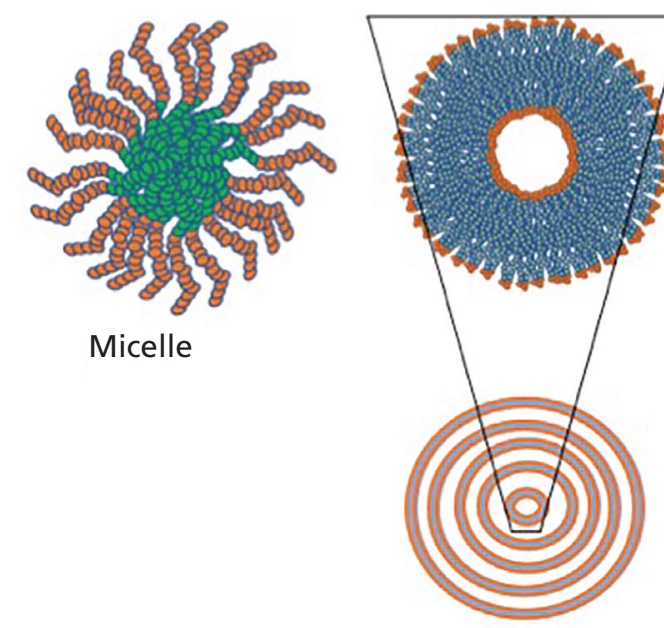
Liposome, in this case multilamellar, where each lamella comprises a bimolecular leaflet with aqueous medium in the centre and between lamellae 
cholesterol, are combined with water. ${ }^{40}$ Each lamella consists of a bimolecular leaflet producing hydrophilic and lipophilic domains that can carry not only lipophilic material but hydrophilic cargo like proteins and peptides, including insulin. ${ }^{41}$ Liposomes can reach $2.5 \mu \mathrm{m}$, comprising single or concentric bilayers, depending on the method of preparation. In high concentration they lose the spherical shape and form an ordered gelatinous tubular mass. Zhang et al recently described an insulin-loaded phospholipid-based phase separation gel that appears to form vesicles, possibly liposomal, during processes releasing the insulin. ${ }^{42}$

\section{Protection}

A gel should offer protection for the fragile insulin from hostile environments of administration sites such as extracellular matrices, gut fluids, etc, and yet foster uptake at the apical surface and be benign in terms of the biological integrity, as described above. ${ }^{8}$ Covalently bonded gels can be enzyme-resistant, the water-filled pores between polymer structuring creating protection against insulin proteolysis because of differential transport for insulin egress and enzyme ingress. It has relevance, particularly for the oral route of insulin delivery, where proteolysis is the main destructive route. ${ }^{43-46}$ The polymer chosen should be compatible with gut $\mathrm{pH}$, but also with the insulin and its native folded state. ${ }^{8}$

Other means exist of enzyme protection by gelation such as the use of enzyme inhibitors. Those containing the YPGA-DTPA conjugate are inherently protective against proteolysis as well as being a structural component, ${ }^{47-49}$ and both albumin and aprotinin have been suggested as sacrificial targets for co-formulated insulin $^{33}$ - for example, in microspheres. ${ }^{50,51}$

\section{Release mechanism: 1 - Basal}

The release mechanisms of dissolved insulin from the protected storage site within a gel include dissolution, diffusion control and, in addition, degradative changes in the polymer ${ }^{52}$ (Figure

Figure 10. Various mechanisms of drug release identified for gels

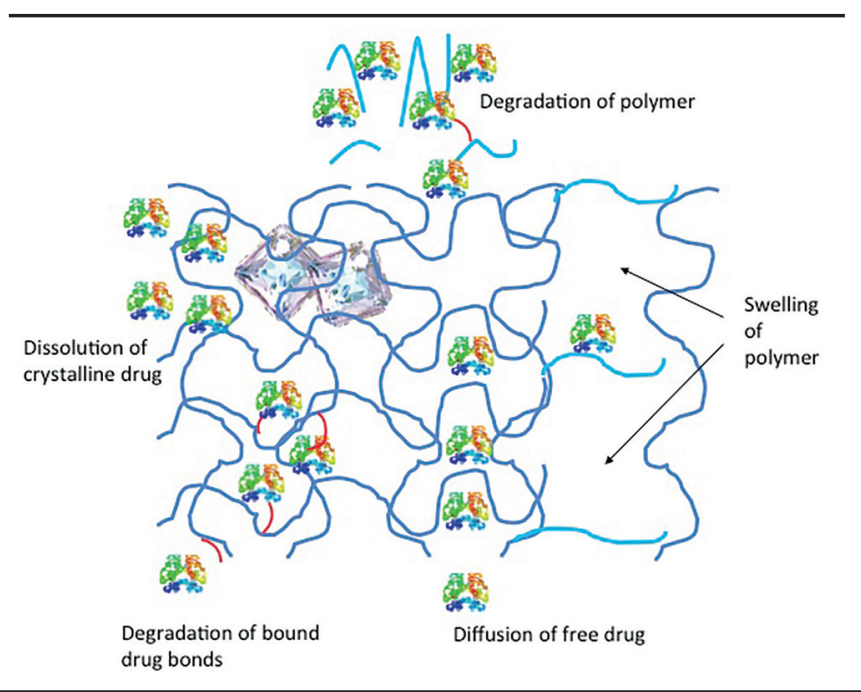

10). For example, a drug release at a sustained basal rate from block polymer gels, bulk gels or hydrogels might simply occur by being able to dissolve or diffuse out, depending on whether it exceeded solubility in the gel solvent when formulated. The kinetics can be zero order (ie, delivery rate is constant) in some circumstances. Alternatively (or additionally), where covalent bonds are involved, release could involve chemically or enzymatically-induced bond breakage to remove obstruction formed by polymer entanglements. While unlikely with a fragile peptide, similar mechanisms could release drug covalently bound to the polymer. ${ }^{53}$

\section{Release mechanism: 2 - Bolus}

For optimally effective diabetes control using gel-administered insulin, the basal dose rate must be supplemented by glucoseresponsive bolus doses that operate automatically and preferably repeatedly to deal quickly and effectively with meal-induced glucose spikes without risk of subsequent hypoglycaemia. A limited triggered system with oral gel responding to gut glucose can hold only the dose appropriate for the daily gut transit time. It would depend on gut glucose content, absorption site and conditions, therefore delivering possibly inappropriate insulin doses for systemic circumstances. Subcutaneous tissue behaves more predictably and implantable gels could hold a larger depot. Skin-based closed loop therefore has advantages, albeit with the known differential with BG and also lacking the hepatocentric delivery of gut insulin.

The methods for formulating insulin to act in a glucoseresponsive way rely on modified or normal insulin formulated with bulk or dispersed carrier materials that interact with the environment to create a differential release rate. Specific and non-specific binding, chelation and charge-related interactions are of particular importance for designs to form the basis of insulin boost dosage in either triggered or closed loop release (in addition to a basal dose rate). ${ }^{54}$ Light, magnetism, electrical signal, salts, $\mathrm{pH}$ and ionic strength mechanisms ${ }^{55-58}$ are all capable of physicochemical stimulus-sensitive design into polymers used for gels for insulin delivery (Figure 11).

The most useful mechanisms would be more clearly glucosespecific, however, so that hyperglycaemia would trigger a prompt and appropriate dose of insulin such that the BG is kept within the target range. The three mechanisms relate to glucose

Figure 11. Innovative designs using physical stresses cause either swelling or shrinking, each of which can result in release

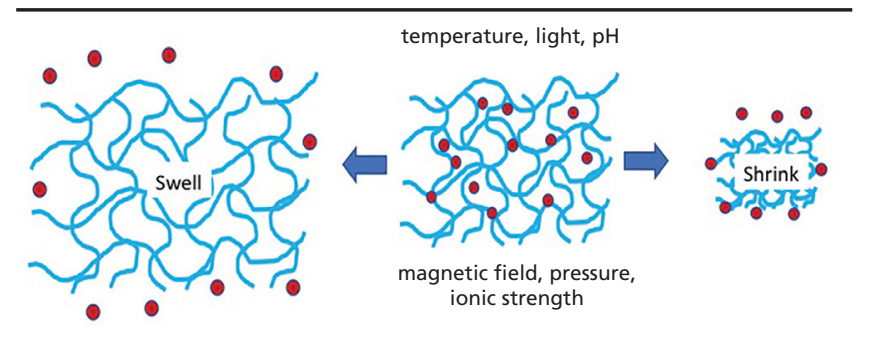


interactions with glucose oxidase, phenylboronic acid and the lectin concanavalin A. These and others have recently been reviewed by Shen et al. ${ }^{59}$

\section{Glucose oxidase (GOD)}

A common mechanism is the $\mathrm{pH}$-linked change that is related to the coupled enzymatic oxidation of glucose to gluconic acid. ${ }^{60}$ For GOD-related mechanisms, the insulin could be a passive passenger or may participate in the mechanism in some way, as exemplified by Fischel-Ghodsian and Newton many years ago in which the solubility of tri-lysyl insulin was manipulated by altering the net charge due to the GOD oxidation. ${ }^{61}$

However, in many cases, a proton-sensitive polymer will either be charged or neutralised as a result and expand to release insulin or contract to expel it (Figure 12a). ${ }^{62} \mathrm{Li}$ et al have described a modification of this in a hydrogel made from a selfassembling peptide with alkaline amino acid side chains that are prevented from linkage when the $\mathrm{pH}$ drops due to the oxidation of glucose. This releases insulin from the loose structure. ${ }^{57} \mathrm{~A}$ recent variation used GOD to modify the aggregation of oppositely charged chitosan-PGLA nano-complexes to release insulin responsively. ${ }^{49}$

Many variations have been devised on the basic model, including liposome types containing GOD, where the polymeric decoration is destabilised by the protons generated to form leaky micelles, thus dispensing insulin in a once-only trigger (Figure 12b). Yu et al have described a sophisticated derivative mechanism of

Figure 12. (a) Glucose oxidase production of protons causes repulsion of polymer changes and thus insulin release. (b) Polymer decoration of liposome collapses when neutralised by protons from glucose oxidation

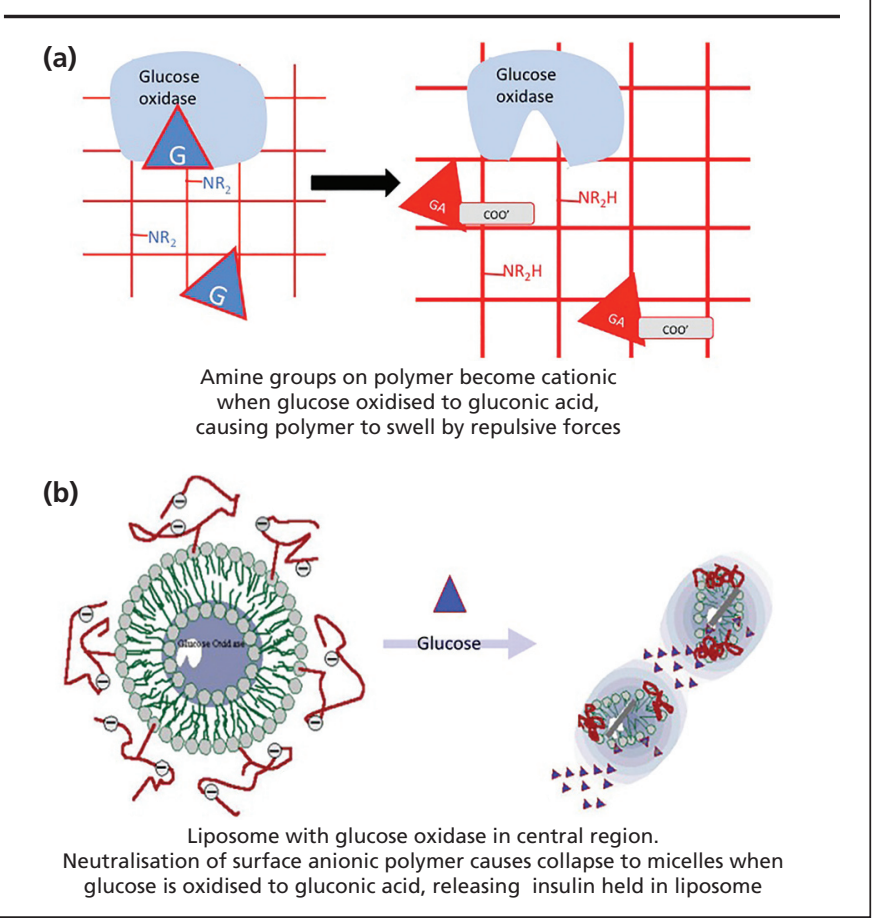

self-assembled micelles comprising cross linked hyaluronic acid with pendant nitroimidazole groups presented in a microneedle array. These undergo a hydrophilic change during the oxygen consumption required to produce gluconic acid (ie, becomes relatively hypoxic). ${ }^{63}$ Alternative nitroimidazole nanoparticulate systems with GOD are described by Jana et $a^{/ 64}$ and in a PEG-polyserine micellar model by Zhao et al. ${ }^{60} \mathrm{~A}$ variation with an alginate carrier cross linked with ferric ions and containing enzyme-coated silica particles fosters the oxidation causing the irreversible degradation of the alginate releasing the insulin. ${ }^{65}$

The GOD-based systems have two serious shortcomings. First is that any local $\mathrm{pH}$ alteration can accomplish the same changes. In addition, the build-up of hydrogen peroxide must be addressed to prevent imposition of a suppressive equilibrium. Catalase has been incorporated to deal with this in simple and complex examples, including a microneedle version that has a conical core containing the insulin as a PVA conjugate plus the enzyme while the shell surrounding it comprises catalase to eliminate peroxide, continuously re-enabling the device but also reducing an inflammatory effect. ${ }^{44,66,67}$

\section{Phenylboronic acid}

Various designs are based on phenylboronic acid (PBA), which interacts by hydrogen bonding to the vicinal $\mathrm{OH}$ or cis-diol moieties of glucose (Figure 13) and can operate in bulk gels, gelatinous particles and vesicles. $68-70$ This is not entirely specific but it is widely discussed for triggered dosing, sometimes by manipulation of the pl. $^{71}$

Li et al describe a hyaluronic acid gel intended to deliver such particles to the enteric apical surface or to be injected subcutaneously. ${ }^{72,73}$ Other nanostructures bear the glucose-sensitive gel-forming material on the exterior surface. ${ }^{74}$

Zhao et al describe insulin in PGLA-PBA microparticles with cross-linking hyaluronic acid as physical bonding to the vicinal $\mathrm{OH}$ groups in the glucuronic acid units. As glucose enters, it displaces the link to occupy the PBA site, loosening the gel structure and allowing insulin to escape. ${ }^{73}$ PBA systems are well enough developed to have been studied for the effects of gamma and neutron irradiation for sterilisation. ${ }^{75}$ Masumoto et al have published work in rodents using a PBA closed loop system. ${ }^{76}$

Minigels with imprinted glucose receptor sites can be formed by polymerising PBA in the presence of glucose, which acts as a template and is then washed out leaving the imprint. Materials like this can behave like lectins, the imprinted pattern imposing a multivalent affinity for glucose. ${ }^{77,78}$

Figure 13. The reversible response of soluble forms of phenylboronic acid to glucose

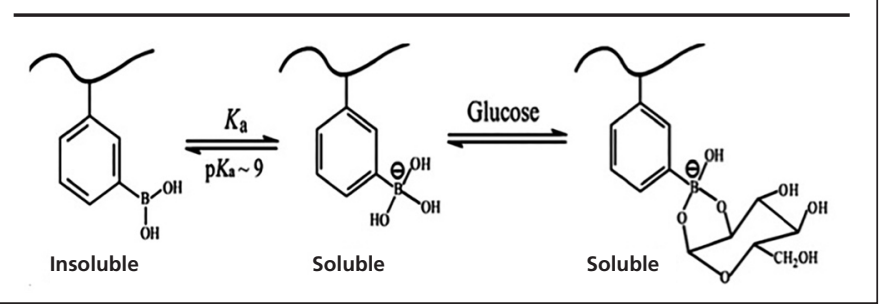




\section{Concanavalin A type}

The lectin con A is a tetrameric protein of MW about $110 \mathrm{kDa}$. Each monomer has a receptor for glucose and several insulin controlling mechanisms have been based on this interaction, including the original Brownlee and Cerami model that had the

Figure 14. Brownlee and Cerami model for competitive displacement of glycosylated insulin by glucose from specific receptors on concanavalin $A$ bound to beads inside a membrane permeable to insulin and glucose

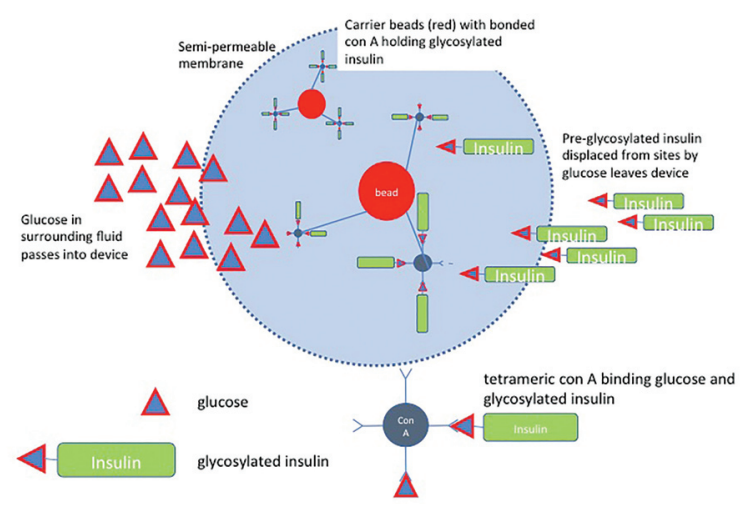

Figure 15. Highly simplistic depictions of the receptor interactions of glucose and concanavalin A

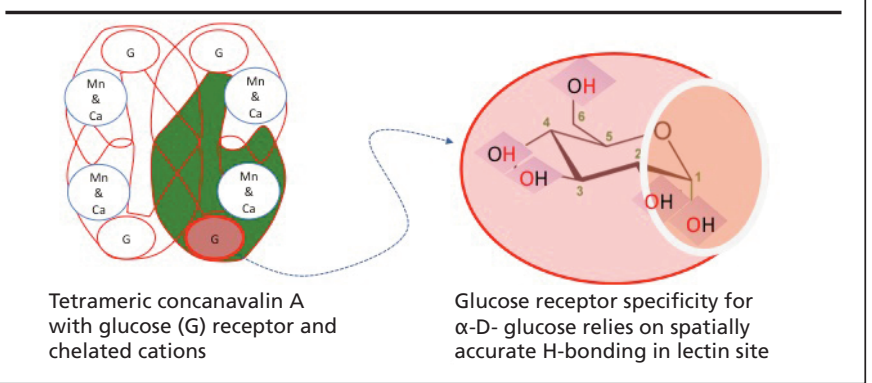

Figure 16. The closed loop system based on gel formation with dextran and concanavalin A, stabilised by light polymerisation. Gel governs the output of insulin from a reservoir delivering to peritoneal tissue

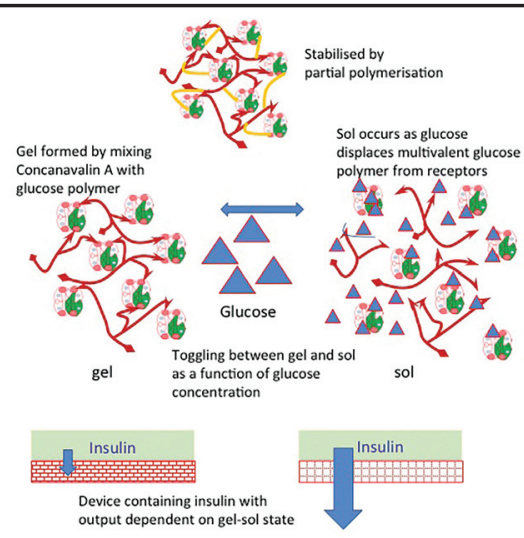

immobilised lectin on beads inside a semipermeable arrangement (Figure 14).

The lectin interacted with insulin glycosylated at the B29 lysine which allowed the insulin to retain activity. ${ }^{79}$ The fully assembled tetrameric protein has four receptor sites for glucose, for which it is fairly specific in terms of human physiological tissue components (Figure 15).

It can interact with glucose itself or saccharides bearing an appropriately configured glucose terminal unit forming a threedimensional network with suitable branched glucans. Nanoparticulate gels have been made from this lectin and a glucan but also from synthetics such as glycosylated acrylate (glucosyloxyethyl methacrylate or GEMA). ${ }^{80}$ Their shrink-swell activity works either as a sensor or as an insulin releasing agent. ${ }^{81}$

A polymerised lectin gel is the basis of an in vivo peritoneal system by Taylor in which insulin output is controlled by a variable viscosity gel from an implantable and refillable reservoir (Figure 16) and extends the insulin implant concept ${ }^{82,83}$ to closed loop. $.33,84-87$

\section{Conclusions}

Gels provide a biocompatible medium for the creative delivery of insulin. They can occur in forms such as semisolids but also as

- Hydrophilic gelatinous formulations are good
candidates to develop into carriers for insulin
- Gels are formed from a variety of materials including
micelle agglomerates, micronised materials and
interpenetrating polymer strands that may be
covalently or physically cross linked to form hydrogels
and minigels
- Gels can be formed from natural and synthetic
polymers, are often biocompatible and frequently
biodegradable
The physicochemical properties of gels make them
able to maintain solubility or accommodate depot
forms of chemically divergent insulin types
Gel formulation of insulin can provide protection
from gut enzymes, providing a means for oral
administration, one advantage of which would be
the provision of hepatocentric delivery
Some polymeric components of gels promote
paracellular uptake of proteins by the gut apical
surface
Polymers and self-assembling particulates are
modifiable in ways that confer properties useful for
interactive functions, that produce potential closed
loop mechanisms responding to ambient glucose
levels


hydrated particulates with viscoelastic properties and a range of structure, size and function, suitable for delivery by several useful routes. This may mean that the treatment of diabetes of both main types may not, in the future, be restricted to subcutaneous injection. Insulin formulation could well comprise gelatinous forms to act as the naked substance or within devices, each creating effects such as hepatocentric, triggered and closed loop delivery. Gels can protect the peptide contents from hostile environments and those with nanostructures may gain apical access or otherwise make routes possible, such as oral. These may be more convenient, increasing compliance and therefore promoting euglycaemia. Closed loop delivery is a further worthy aim where the insulin dose is tailored closely to changes in the BG. Presently, closed loop delivery is experimental and either biologically or electronically controlled. Neither of these is in general use and both still have significant drawbacks. Development of gel systems is currently also addressing the closed loop aim and, with the polymer synthesis variety available, seems worth the effort to offer an efficient counter to the complications of diabetes.

\section{Conflict of interest None. Funding None.}

\section{References}

1. Joslin EP. The treatment of diabetes mellitus. Can Med Assoc J 1916; 6(8):673-84.

2. Home PD. Impact of the UKPDS--an overview. Diabet Med 2008;25(Suppl 2):2-8.

3. Nathan DM. The diabetes control and complications trial/epidemiology of diabetes interventions and complications study at 30 years: overview. Diabetes Care 2014;37(1):9-16. https://doi.org/10.2337/dc13-2112.

4. de Lusignan S, Hinton W, Konstantara E, et al. Intensification to injectable therapy in type 2 diabetes: Mixed methods study (protocol). BMC Health Serv Res 2019;19(1):284-94. https://doi.org/10.1186/s12913-019-4112-3.

5. Bloomgarden Z. Is insulin the preferred treatment for $\mathrm{HbA} 1 \mathrm{c}>9 \%$ ? J Diabetes 2017;9(9):814-6. https://doi.org/10.1111/1753-0407.12575.

6. Sarbacker GB, Urteaga EM. Adherence to insulin therapy. Diabetes Spectrum 2016;29(3):166-70. https://doi.org/10.2337/diaspect.29.3.166.

7. Weiss MA, Lawrence MC. A thing of beauty: structure and function of insulin's "aromatic triplet". Diabetes Obes Metab 2018;20(Suppl 2):51-63. https://doi.org/10.1111/dom.13402.

8. Nur M, Vasiljevic T. Can natural polymers assist in delivering insulin orally? Int J Biol Macromol 2017;103:889-901. https://doi.org/S0141-8130(17)30213-1 [pii].

9. Chaturvedi K, Ganguly K, Nadagouda MN, Aminabhavi TM. Polymeric hydrogels for oral insulin delivery. J Controlled Release 2013;165(2):129-38. https://doi.org/10.1016/j.jconrel.2012.11.005.

10. Zare Y, Rhee KY. Prediction of loss factor $(\tan \delta)$ for polymer nanocomposites as a function of yield tress, relaxation time and the width of transition region between newtonian and power-law behaviors. J Mechanical Behavior Biomed Materials 2019;96:136-43. https://doi.org/10.1016/j.jmbbm.2019.04.045

11. Jacob J, Haponiuk JT, Thomas S, Gopi S. Biopolymer based nanomaterials in drug delivery systems: a review. Materials Today Chemistry 2018;9:43-55. https://doi.org/10.1016/j.mtchem.2018.05.002.

12. Yang G, Wang Q, Gao Y, Yang C, Hu L. Combination of coating and injectable hydrogel depot to improve the sustained delivery of insulin. J Drug Delivery Sci Technol 2018;45:415-21. https://doi.org/10.1016/j.jddst.2018.03.028.

13. Bodratti AM, Alexandridis P. Amphiphilic block copolymers in drug delivery: advances in formulation structure and performance. Expert Opin Drug Deliv 2018;15(11):1085-104. https://doi.org/10.1080/17425247.2018.1529756.

14. Taylor MJ, Tomlins P, Sahota ST. Thermoresponsive gels. Gels 2017;3(1). https://doi.org/10.3390/gels3010004.
15. Choi S, Kim SW. Controlled release of insulin from injectable biodegradable triblock copolymer depot in ZDF rats. Pharm Res 2003;20(12):2008-10. https://doi.org/10.1023/b:pham.0000008050.99985.5c.

16. Chen $Y$, Li Y, Shen $W$, et al. Controlled release of liraglutide using thermogelling polymers in treatment of diabetes. Sci Rep 2016;6:31593. https://doi.org/10.1038/srep31593.

17. Sharma G, Sharma AR, Nam J, Doss GPC, Lee S, Chakraborty C. Nanoparticle based insulin delivery system: the next generation efficient therapy for type 1 diabetes. J Nanobiotechnology 2015;13:74. https://www.ncbi. nlm.nih.gov/pubmed/26498972; https://www.ncbi.nlm.nih.gov/pmc/articles/PMC4619439/. https://doi.org/10.1186/s12951-015-0136-y.

18. Borzacchiello A, Russo L, Malle BM, Schwach-Abdellaoui K, Ambrosio L. Hyaluronic acid based hydrogels for regenerative medicine applications. Biomed Res Int 2015;2015:871218. https://doi.org/10.1155/2015/871218.

19. Caló E, Khutoryanskiy VV. Biomedical applications of hydrogels: a review of patents and commercial products. Eur Polymer J 2015;65:252-67. https://doi.org/10.1016/j.eurpolymj.2014.11.024.

20. Ionov L. Hydrogel-based actuators: possibilities and limitations. Materials Today 2014;17(10):494-503. https://doi.org/10.1016/j.mattod.2014.07.002.

21. Bae JW, Choi JH, Lee Y, Park KD. Horseradish peroxidase-catalysed in situforming hydrogels for tissue-engineering applications. J Tissue Eng Regen Med 2015;9(11):1225-32. https://doi.org/10.1002/term.1917.

22. Nguyen DT, Phan VHG, Lee DS, Thambi T, Huynh DP. Bioresorbable pH- and temperature-responsive injectable hydrogels-incorporating electrosprayed particles for the sustained release of insulin. Polym Degrad Stab 2019;162:36-46. https://doi.org/10.1016/j.polymdegradstab.2019.02.013.

23. Custodio CA, Reis RL, Mano JF. Photo-cross-linked laminarin-based hydrogels for biomedical applications. Biomacromolecules 2016;17(5):1602-09. https://doi.org/10.1021/acs.biomac.5b01736.

24. El-Sherbiny I, Yacoub MH. Hydrogel scaffolds for tissue engineering: Progress and challenges. Global Cardiol Sci Pract 2013;2013(3):316-42. https://doi.org/10.5339/gcsp.2013.38.

25. Bai X, Gao M, Syed S, Zhuang J, Xu X, Zhang X. Bioactive hydrogels for bone regeneration. Bioactive Materials 2018;3(4):401-17. https://doi.org/10.1016/j.bioactmat.2018.05.006.

26. Arciola C, Campoccia D, Montanaro L. Implant infections: Adhesion, biofilm formation and immune evasion. Volume 16, 2018. https://doi.org/10.1038/s41579-018-0019-y.

27. Mallawarachchi S, Mahadevan A, Gejji V, Fernando S. Mechanics of controlled release of insulin entrapped in polyacrylic acid gels via variable electrical stimuli. Drug Deliv Trans/ Res 2019;9(4):783-94.

https://doi.org/10.1007/s13346-019-00620-7.

28. Zhou T, Ding L, Che G, Jiang W, Sang L. Recent advances and trends of molecularly imprinted polymers for specific recognition in aqueous matrix: Preparation and application in sample pretreatment. TrAC Trends in Analytical Chemistry 2019;114:11-28. https://doi.org/10.1016/j.trac.2019.02.028.

29. Arifuzzaman MD, Zhao W, Zhao Y. Surface ligands in the imprinting and binding of molecularly imprinted cross-linked micelles. Supramol Chem 2018;30(11):929-39. https://doi.org/10.1080/10610278.2018.1489540.

30. Yao $Y$, Wei $Y$, Chen $S$. Size effect of the surface energy density of nanoparticles. Surface Sci 2015;636:19-24. https://doi.org/10.1016/j.susc.2015.01.016.

31. Han L, Zhao Y, Yin L, et al. Insulin-loaded pH-sensitive hyaluronic acid nanoparticles enhance transcellular delivery. AAPS PharmSciTech 2012;13(3):836-45. https://doi.org/10.1208/s12249-012-9807-2.

32. Arbit E, Kidron M. Oral insulin delivery in a physiologic context: review. J Diabetes Sci Technol 2017:11(4):825-32. https://doi.org/10.1177/1932296817691303.

33. Hu Q, Luo Y. Recent advances of polysaccharide-based nanoparticles for oral insulin delivery. Int J Biol Macromol 2018;120:775-82. https://doi.org/10.1016/j.ijbiomac.2018.08.152.

34. Mukhopadhyay P, Mishra R, Rana D, Kundu PP. Strategies for effective oral insulin delivery with modified chitosan nanoparticles: a review. Progress in Polymer Sci 2012;37(11):1457-75. https://doi.org/10.1016/.jprogpolymsci.2012.04.004.

35. Fonte P, Araújo F, Silva C, et al. Polymer-based nanoparticles for oral insulin delivery: Revisited approaches. Biotechnol Adv 2015;33(6, Part 3):134254. https://doi.org/10.1016/j.biotechadv.2015.02.010.

36. Alai MS, Lin WJ, Pingale SS. Application of polymeric nanoparticles and mi- 
celles in insulin oral delivery. J Food Drug Anal 2015;23(3):351-8. https://doi.org/10.1016/j.jfda.2015.01.007.

37. Mumuni MA, Kenechukwu FC, Ofokansi KC, Attama AA, Díaz DD. Insulinloaded mucoadhesive nanoparticles based on mucin-chitosan complexes for oral delivery and diabetes treatment. Carbohydr Polym 2020; 229:115506. https://doi.org/10.1016/j.carbpol.2019.115506.

38. Sharma D, Singh J. Long-term glycemic control and prevention of diabetes complications in vivo using oleic acid-grafted-chitosan zinc-insulin complexes incorporated in thermosensitive copolymer. I Controlled Release 2020;323:161-78. https://doi.org/10.1016/j.jconrel.2020.04.012

39. Sudhakar S, Chandran SV, Selvamurugan N, Nazeer RA. Biodistribution and pharmacokinetics of thiolated chitosan nanoparticles for oral delivery of insulin in vivo. Int J Biol Macromol 2020;150:281-8. https://doi.org/10.1016/j.ijbiomac.2020.02.079.

40. Wong CY, Al-Salami H, Dass CR. Recent advancements in oral administration of insulin-loaded liposomal drug delivery systems for diabetes mellitus. Int $J$ Pharm 2018;549(1):201-7. https://doi.org/10.1016/j.jpharm.2018.07.041.

41. Mohsen AM. Nanotechnology advanced strategies for the management of diabetes mellitus. Curr Drug Targets 2019;20(10):995-1007 https://doi.org/10.2174/1389450120666190307101642.

42. Zhang T, Luo J, Peng Q, et al. Injectable and biodegradable phospholipidbased phase separation gel for sustained delivery of insulin. Colloids and Surfaces B: Biointerfaces 2019;176:194-201. https://doi.org/10.1016/j.colsurfb.2019.01.003.

43. Yang J, Cao Z. Glucose-responsive insulin release: analysis of mechanisms, formulations, and evaluation criteria. J Controlled Release 2017:263:2319. https://doi.org/10.1016/j.jconrel.2017.01.043

44. Wang J, Ye Y, Yu J, et al. Core-shell microneedle gel for self-regulated insulin delivery. ACS Nano 2018;12(3):2466-73. https://doi.org/10.1021/acsnano.7b08152

45. Woo VC. New insulins and new aspects in insulin delivery. Can J Diabetes 2015;39(4):335-43. http://dx.doi.org/10.1016/j.jcjd.2015.04.006

46. Priya James $H$, John $R$, Alex A, Anoop KR. Smart polymers for the controlled delivery of drugs - a concise overview. Acta Pharm Sin B 2014;4(2):120-7. https://doi.org/10.1016/j.apsb.2014.02.005.

47. Su FY, Lin KJ, Sonaje K, et al. Protease inhibition and absorption enhancement by functional nanoparticles for effective oral insulin delivery. Biomaterials 2012;33(9):2801-11. https://doi.org/10.1016/..biomaterials.2011.12.038.

48. Chuang EY, Lin KJ, Su FY, et al. Calcium depletion-mediated protease inhibition and apical-junctional-complex disassembly via an EGTA-conjugated carrier for oral insulin delivery. J Control Release 2013;169(3):296-305. https://doi.org/10.1016/j.jconrel.2012.11.011.

49. Mohammadpour F, Hadizadeh F, Tafaghodi M, et al. Preparation, in vitro and in vivo evaluation of PLGA/chitosan based nano-complex as a novel insulin delivery formulation. Int J Pharm 2019;572:118710. https://doi.org/10.1016/j.jpharm.2019.118710.

50. Agrawal G, Wakte P, Shelke $S$. Formulation optimization of human insulin loaded microspheres for controlled oral delivery using response surface methodology. Endocr Metab Immune Disord Drug Targets 2017; 17(2):149-65. https://doi.org/10.2174/1871530317666170503120129.

51. Baykan O, Yaman A, Gerin F, Sirikci O, Haklar G. The effect of different protease inhibitors on stability of parathyroid hormone, insulin, and prolactin levels under different lag times and storage conditions until analysis. J Clin Lab Anal 2017:31(6). Epub 30 Jan 2017. https://doi.org/10.1002/jcla.22144.

52. Hu J, Chen Y, Li Y, Zhou Z, Cheng Y. A thermo-degradable hydrogel with light-tunable degradation and drug release. Biomaterials 2017:112:13340. https://doi.org/10.1016/j.biomaterials.2016.10.015.

53. Sun DD, Lee PI. Crosslinked hydrogels-a promising class of insoluble solid molecular dispersion carriers for enhancing the delivery of poorly soluble drugs. Acta Pharm Sin B 2014;4(1):26-36.

https://doi.org/10.1016/j.apsb.2013.12.002.

54. Ferreira NN, Ferreira LMB, Cardoso VMO, Boni Fl, Souza ALR, Gremião MPD. Recent advances in smart hydrogels for biomedical applications: from selfassembly to functional approaches. Eur Polymer J 2018;99:117-33. https://doi.org/10.1016/j.eurpolymj.2017.12.004.

55. Lin Y, Mi F, Lin $\mathrm{P}$, et al. Strategies for improving diabetic therapy via alternative administration routes that involve stimuli-responsive insulin-delivering systems. Adv Drug Deliv Rev 2019;139:71-82. https://doi.org/10.1016/j.addr.2018.12.001

56. Lim H, Ooi C, Tey B, Chan E. Controlled delivery of oral insulin aspart using $\mathrm{pH}$-responsive alginate/K-carrageenan composite hydrogel beads. React Funct Polym 2017;120:20-9.

https://doi.org/10.1016/j.reactfunctpolym.2017.08.015.

57. Li X, Fu M, Wu J, et al. pH-sensitive peptide hydrogel for glucose-responsive insulin delivery. Acta Biomaterialia 2017;51:294-303. https://doi.org/10.1016/j.actbio.2017.01.016.

58. Nguyen DT, Phan VHG, Lee DS, Thambi T, Huynh DP. Bioresorbable pH- and temperature-responsive injectable hydrogels-incorporating electrosprayed particles for the sustained release of insulin. Polym Degrad Stab 2019:162:36-46. https://doi.org/10.1016/j.polymdegradstab.2019.02.013.

59. Shen $D, Y u H$, Wang $L$, et al. Recent progress in design and preparation of glucose-responsive insulin delivery systems. I Controlled Release 2020;321:236-58. https://doi.org/10.1016/j.jconrel.2020.02.014.

60. Zhao L, Wang L, Zhang Y, et al. Glucose oxidase-based glucose-sensitive drug delivery for diabetes treatment. Polymers (Basel) 2017;9(7):255-76. https://doi.org/10.3390/polym9070255.

61. Fischel-Ghodsian F, Newton JM. Simulation and optimisation of a selfregulating insulin delivery system. J Drug Target 1993;1(1):67-80. https://doi.org/10.3109/10611869308998766.

62. Peppas N, Bures, Christie. Glucose-responsive hydrogels. Volume 112, 2008. https://doi.org/10.1201/b18990-114.

63. Yu J, Zhang Y, Ye Y, et al. Microneedle-array patches loaded with hypoxiasensitive vesicles provide fast glucose-responsive insulin delivery. Proc Natl Acad Sci USA 2015;112(27):8260-5. https://doi.org/10.1073/pnas.1505405112.

64. Jana BA, Shinde U, Wadhwani A. Preparation of enzyme based polymeric biomimetic nanoparticle for the controlled release of insulin. Sensing and Bio-Sensing Res 2020;28:100342. https://doi.org/10.1016/j.sbsr.2020.100342.

65. Scheja S, Domanskyi S, Gamella M, et al. Glucose-triggered insulin release from Fe3+-cross-linked alginate hydrogel: experimental study and theoretical modeling. ChemPhysChem 2017;18(12):1541-51. https://doi.org/10.1002/cphc.201700195.

66. Zhang Y, Yu J, Kahkoska AR, Wang J, Buse JB, Gu Z. Advances in transdermal insulin delivery. Adv Drug Deliv Rev 2019;139:51-70. https://doi.org/10.1016/j.addr.2018.12.006.

67. Luo J, Cao S, Chen X, et al. Super long-term glycemic control in diabetic rats by glucose-sensitive LbL films constructed of supramolecular insulin assembly. Biomaterials 2012;33(33):8733-42. https://doi.org/10.1016/ j.biomaterials.2012.08.041.

68. Wu J, Bremner DH, Li H, Sun X, Zhu L. Synthesis and evaluation of temperature- and glucose-sensitive nanoparticles based on phenylboronic acid and $\mathrm{N}$-vinylcaprolactam for insulin delivery. Materials Science and Engineering: C 2016;69:1026-35. https://doi.org/10.1016/j.msec.2016.07.078.

69. Wu J, Bremner DH, Li H, Niu S, Li S, Zhu L. Phenylboronic acid-diol crosslinked 6-O-vinylazeloyl-d-galactose nanocarriers for insulin delivery. Materials Science and Engineering: C 2017;76:845-55. https://doi.org/10.1016/..msec.2017.03.139.

70. Joseph VS, Hong J. Phenylboronic acid-modified oligoamine sensitive to monosaccharides and carbon dioxide under physiological conditions. Colloids and Surfaces A: Physicochemical and Engineering Aspects 2018; 553:312-6. https://doi.org/10.1016/j.colsurfa.2018.05.084.

71. Nguyen T, Magda JJ, Tathireddy P. Manipulation of the isoelectric point of polyampholytic smart hydrogels in order to increase the range and selectivity of continuous glucose sensors. Sensors and Actuators B: Chemical 2018; 255:1057-63. https://doi.org/10.1016/j.snb.2017.08.022.

72. Li L, Jiang G, Yu W, et al. A composite hydrogel system containing glucoseresponsive nanocarriers for oral delivery of insulin. Materials Science and Engineering: C 2016;69:37-45. https://doi.org/10.1016/..msec.2016.06.059.

73. Zhao F, Wu D, Yao D, et al. An injectable particle-hydrogel hybrid system for glucose-regulatory insulin delivery. Acta Biomaterialia 2017;64:334-45. https://doi.org/10.1016/j.actbio.2017.09.044.

74. Gao W, Hu Y, Xu L, Liu M, Wu H, He B. Dual pH and glucose sensitive gel gated mesoporous silica nanoparticles for drug delivery. Chinese Chemical Letters 2018;29(12):1795-8. https://doi.org/10.1016/j.cclet.2018.05.022.

75. Magda J, Cho S, Streitmatter S, Jevremovic T. Effects of gamma rays and neutron irradiation on the glucose response of boronic acid-containing "smart" hydrogels. Polym Degrad Stab 2014;99:219-22. https://doi.org/10.1016/j.polymdegradstab.2013.11.002. 
76. Matsumoto A, Tanaka M, Matsumoto H, et al. Synthetic "smart gel" provides glucose-responsive insulin delivery in diabetic mice. Sci Adv 2017; 3(11):eaaq0723. https://doi.org/110.1126/sciadv.aaq0723.

77. Byrne ME, Park K, Peppas NA. Molecular imprinting within hydrogels. Advanced Drug Delivery Rev 2002;54(1):149-61. https://doi.org/10.1016/S0169-409X(01)00246-0.

78. Peng M, Xiang H, Hu X, Shi S, Chen X. Boronate affinity-based surface molecularly imprinted polymers using glucose as fragment template for excellent recognition of glucosides. J Chromatogr A 2016;1474:8-13. https://doi.org/ 10.1016/i.chroma.2016.10.059.

79. Brownlee M, Cerami A. A glucose-controlled insulin-delivery system: Semisynthetic insulin bound to lectin. Science 1979;206(4423):1190-1. https://doi.org/10.1126/science.505005.

80. Yin R, Bai M, He J, Nie J, Zhang W. Concanavalin A-sugar affinity based system: Binding interactions, principle of glucose-responsiveness, and modulated insulin release for diabetes care. Int J Biol Macromol 2019;124:72432. https://doi.org/10.1016/j.ijbiomac.2018.11.261.

81. Kawamura A, Hata Y, Miyata T, Uragami T. Synthesis of glucose-responsive bioconjugated gel particles using surfactant-free emulsion polymerization. Colloids Surf B Biointerfaces 2012;99:74-81.

https://doi.org/10.1016/j.colsurfb.2011.10.008.
82. Park YS. Novel route of insulin delivery using an implant-mediated drug delivery system. Drug Deliv Trans/ Res 2017;7(2):286-91. https://doi.org/10.1007/s13346-016-0354-3.

83. Rieger C, Kurz K, Mueller-Hoffmann W, Gehr B, Liebl A. New design of a percutaneous port system for continuous intraperitoneal insulin infusion. J Diabetes Sci Technol 2019:13:1158-60. https://doi.org/10.1177/1932296819855425.

84. Taylor MJ, Tanna S, Sahota TS, Sawicka K. Closed loop glucose control of diabetic rats. J Pharmacy Pharmacol 2007;59(S1):A15.

85. Taylor MJ, Tanna S, Sahota T. In vivo study of a polymeric glucose-sensitive insulin delivery system using a rat model. J Pharm Sci 2010;99(10):421527. https://doi.org/10.1002/jps.22138.

86. Taylor MJ, Gregory R, Tomlins P, Jacob D, Hubble J, Sahota TS. Closed-loop glycaemic control using an implantable artificial pancreas in diabetic domestic pig (Sus scrofa domesticus). Int J Pharm 2016;500(1-2):371-8. https://doi.org/10.1016/j.jpharm.2015.12.024.

87. Lin K, Yi J, Mao X, Wu H, Zhang L, Yang L. Glucose-sensitive hydrogels from covalently modified carboxylated pullulan and concanavalin A for smart controlled release of insulin. Reactive and Functional Polymers 2019;139:1129. https://doi.org/10.1016/j.reactfunctpolym.2019.01.016. 